\title{
СТРУКТУРА ОБЛАСТЕЙ РЕГУЛЯРНОГО ДВИЖЕНИЯ В ФАЗОВОМ ПРОСТРАНСТВЕ КАНАЛИРОВАННЫХ ЭЛЕКТРОНОВ
}

\author{
(С) 2020 г. В. В. Сыщенко ${ }^{a,}$, А. И. Тарновский ${ }^{a}$, А. Ю. Исупов ${ }^{b}$, И. И. Соловьев ${ }^{a}$ \\ ${ }^{a}$ Белгородский государственный национальный исследовательский университет, \\ Белгород, 308015 Россия \\ ${ }^{b}$ Лаборатория физики высоких энергий ОИЯИ, Дубна, 141980 Россия \\ *e-mail: syshch@yandex.ru \\ Поступила в редакцию 26.07.2019 г. \\ После доработки 30.08.2019 г. \\ Принята к публикации 31.08.2019 г.
}

\begin{abstract}
Движение электрона в режиме аксиального каналирования в направлении [100] кристалла кремния может быть как регулярным, так и хаотическим (в зависимости от начальных условий). В статье найден вклад областей регулярной и хаотической динамики в квазиклассическую плотность уровней энергии поперечного движения электронов. Полученные значения использованы в качестве параметров распределения Берри-Робника, описывающего статистику межуровневых расстояний в случае сосуществования областей регулярного и хаотического движения.
\end{abstract}

Ключевые слова: регулярная динамика, хаотическая динамика, квантовый хаос, каналирование, квазиклассическое приближение, статистика межуровневых расстояний, распределение БерриРобника.

DOI: $10.31857 / \mathrm{S} 1028096020030188$

\section{ВВЕДЕНИЕ}

Проблематика квантового хаоса означает исследование отличий в поведении квантовых систем, обладающих в классическом пределе хаотической динамикой, с одной стороны, и обладающих регулярной динамикой, с другой стороны [1-4]. Одним из самых простых для анализа проявлений квантового хаоса являются статистические свойства уровней энергии квантовой системы. Так, расстояние $s$ между соседними уровнями энергии хаотической системы подчиняется распределению Вигнера [2-4]:

$$
p(s)=\left(\pi \rho^{2} s / 2\right) \exp \left(-\pi \rho^{2} s^{2} / 4\right),
$$

где $\rho-$ средняя плотность уровней на рассматриваемом участке энергетического спектра системы, в то время как для систем с регулярной динамикой имеет место экспоненциальное (пуассоновское) распределение:

$$
p(s)=\rho \exp (-\rho s),
$$

с максимумом при $s=0$.

Таким образом, в регулярных системах имеет место тенденция к группированию уровней энергии в оболочки, в то время как в хаотических системах уровни энергии проявляют тенденцию к взаимному отталкиванию. Поэтому проявления квантового хаоса наиболее заметны в квазиклас- сической области, где количество энергетических уровней велико.

Проявления динамического хаоса в каналировании электронов $[5,6]$ исследовались в работах [7-9] для случая движения вблизи направления [110] кристалла кремния. В этом случае пары соседних атомных цепочек создают двухъямный потенциал, выше седловой точки которого движение электронов оказывается почти полностью хаотическим. Было установлено, что статистические свойства уровней в этой области хорошо описываются распределением Вигнера (1).

Более сложным является случай, когда классическая динамика частицы оказывается при заданной энергии регулярной для одних начальных условий и хаотической для других. Такая ситуация реализуется, например, при движении электрона вблизи направления [100] кристалла кремния [6]. Предполагая, что в квазиклассическом пределе области регулярного движения и (единственная) область хаотического движения порождают две независимые последовательности уровней, Берри и Робник [10], а также (независимо) Богомольный [11] показали, что распределение межуровневых расстояний будет описываться следующей формулой: 


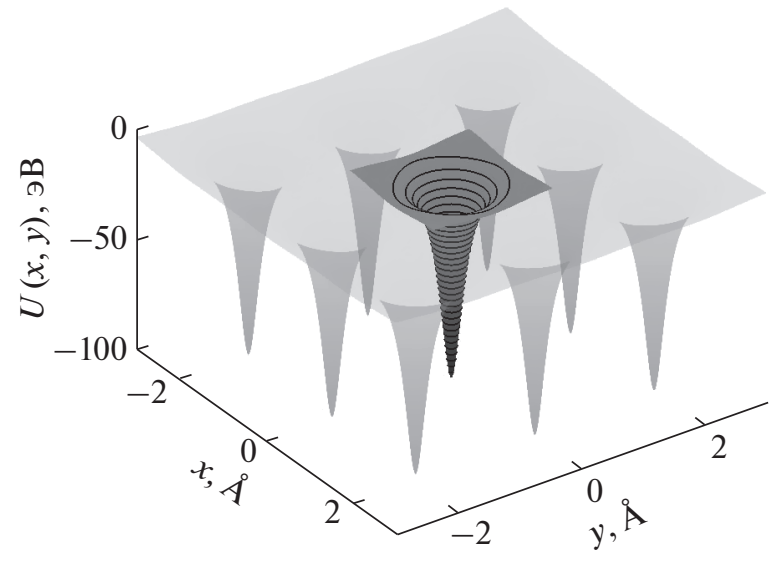

Рис. 1. Потенциальная энергия (10) электрона в поле цепочки [100] кристалла кремния с учетом вкладов восьми ее ближайших соседей.

$$
\begin{gathered}
p(s)=\frac{1}{\rho} \exp \left(-\rho_{1} s\right)\left(\rho_{1}^{2} \operatorname{erfc}\left(\pi^{1 / 2} \rho_{2} s / 2\right)+\right. \\
\left.+\left(2 \rho_{1} \rho_{2}+\pi \rho_{2}^{3} s / 2\right) \exp \left(-\pi \rho_{2}^{2} s^{2} / 4\right)\right),
\end{gathered}
$$

где $\rho_{1}$ и $\rho_{2}-$ плотности уровней, обусловленных регулярной и хаотической динамикой, соответственно $\left(\rho_{1}+\rho_{2}=\rho\right)$,

$$
\operatorname{ercf}(x)=2 \pi^{-1 / 2} \int_{x}^{\infty} \exp \left(-t^{2}\right) d t=1-\operatorname{erf}(x) .
$$

Средняя плотность уровней энергии двумерной системы в квазиклассическом пределе квантовой механики определяется формулой [10]:

$$
\rho(E)=(2 \pi \hbar)^{-2} \int \delta\left(E-H\left(x, y, p_{x}, p_{y}\right)\right) d x d y d p_{x} d p_{y},(4)
$$

где $H$ - классический гамильтониан системы, а дельта-функция выражает закон сохранения энергии при движении частицы. Выполняя интегрирование по $p_{y}$, получим

$$
\rho(E)=2(2 \pi \hbar)^{-2} \int\left|v_{y}\left(x, y, p_{x}\right)\right|^{-1} d x d y d p_{x},
$$

где $v_{y}-$ компонента скорости частицы в направлении оси $y$, а интегрирование ведется по области, определяемой условием

$$
p_{x}^{2} / 2 m+U(x, y) \leq E .
$$

В настоящей статье выполнена оценка вкладов $\rho_{1}$ и $\rho_{2}$ в среднюю плотность невырожденных уровней энергии (5), основанная на учете структуры областей регулярного движения в фазовом пространстве электрона, движущегося вблизи направления [100] кристалла кремния в режиме аксиального каналирования.

\section{МЕТОДИКА}

Если релятивистская частица падает под малым углом $\psi$ к плотно упакованной атомами кристаллографической оси, то возможна ситуация, когда она будет совершать финитное движение в поперечной (по отношению к оси) плоскости, называемое аксиальным каналированием [5, 6]. В этом случае движение частицы может быть описано как движение в непрерывном потенциале атомной цепочки, усредненном вдоль ее длины. В таком потенциале сохраняется компонента импульса частицы $p_{\|}$, параллельная оси цепочки, что позволяет описывать движение электрона в поперечной плоскости с помощью двумерного уравнения Шредингера

$$
\widehat{H} \Psi(x, y, t)=i \hbar \frac{\partial}{\partial t} \Psi(x, y, t)
$$

с оператором Гамильтона

$$
\widehat{H}=-\frac{\hbar^{2}}{2 E_{\|} / c^{2}}\left(\frac{\partial^{2}}{\partial x^{2}}+\frac{\partial^{2}}{\partial y^{2}}\right)+U(x, y),
$$

в котором роль массы частицы играет величина $E_{\|} / c^{2}$, где $E_{\|}=\left(m^{2} c^{4}+p_{\|} c^{2}\right)^{1 / 2}-$ энергия продольного движения [5].

Непрерывный потенциал отдельной атомной цепочки может быть аппроксимирован формулой [5]:

$$
U_{1}(x, y)=-U_{0} \ln \left(1+\frac{\beta R^{2}}{x^{2}+y^{2}+\alpha R^{2}}\right),
$$

где для цепочки [100] кристалла кремния $U_{0}=66.6$ эВ, $\alpha=0.48, \beta=1.5, R=0.194 \AA$ (радиус Томаса-Ферми). Такие цепочки образуют в плоскости (100) квадратную решетку с периодом $a \approx 1.92 \AA$ А̊. Потенциальная энергия электрона в поле цепочки с учетом вкладов восьми ее ближайших соседей будет описываться суммой:

$$
U(x, y)=\sum_{i=-1}^{1} \sum_{j=-1}^{1} U_{1}(x-i a, y-j a),
$$

вид этой функции приведен на рис. 1.

Собственные значения $E_{\perp}$ гамильтониана (8) с потенциалом (10) (уровни энергии поперечного движения каналированного электрона) находятся численно с помощью так называемого спектрального метода, описанного в [7, 8, 12, 13]. Отметим, что в рассматриваемом случае потенциал будет обладать симметрией квадрата, и все доступные для частицы состояния можно классифицировать по неприводимым представлениям группы $D_{4}$ (или изоморфной ей $C_{4 v}$, см., например, [14]) в зависимости от типа симметрии волновой функции. Эта группа имеет четыре одномерных неприводимых представления, соответствующих невырожденным уровням энергии, и 
одно двумерное, соответствующее двукратно вырожденным уровням.

Классическая динамика электрона в потенциале (10) исследуется методом сечений Пуанкаре [3-6]. Напомним, что регулярность либо хаотичность движения частицы тесно связана с интегрируемостью уравнения движения. Если число интегралов движения равно числу степеней свободы (в нашем случае - двум), система является интегрируемой. Траектория частицы в фазовом пространстве будет в этом случае лежать на поверхности:

$$
E_{\perp}=H\left(x, y, p_{x}, p_{y}\right),
$$

обладающей топологическими свойствами тора. Движение в этом случае оказывается регулярным (периодическим либо квазипериодическим). В противном случае движение будет хаотическим.

Метод сечений Пуанкаре позволяет выяснить наличие у системы еще одного (наряду с энергией) интеграла движения. В этом методе в процессе численного моделирования траектории частицы отмечаются все точки пересечения траектории с какой-либо плоскостью в фазовом пространстве, например, с плоскостью $\left(x, p_{x}\right)$. Если, наряду с энергией, существует еще некоторый интеграл движения:

$$
J=J\left(x, y, p_{x}, p_{y}\right),
$$

то, исключая из уравнений (11) и (12) переменную $p_{y}$ при условии $y=0$, мы получим связь между переменными $x$ и $p_{x}$. На графике Пуанкаре в этом случае отмечаемые точки будут ложиться на плавную кривую (рис. 2). В случае отсутствия второго интеграла движения точки будут ложиться хаотическим образом в пределах некоторой области (как, например, на рис. 2б).

Вклад областей регулярного движения в полную плотность уровней (4) оценивался следующим образом. При вычислении интеграла (5) методом Монте-Карло случайные точки $\left(x, y, p_{x}\right)$, попавшие в разрешенную для движения область (6), рассматривались как начальные точки фазовых траекторий, каждая из которых прослеживалась до пересечения с плоскостью $\left(x, p_{x}\right)$. В случае, если точка пересечения попадала внутрь области регулярного движения на сечении Пуанкаре, вклад соответствующей начальной точки учитывался не только в полной величине плотности уровней $\rho$, но и в величине плотности $\rho_{1}$, соответствующей регулярному движению. Подчеркнем, что для заданной начальной точки $\left(x, y, p_{x}\right)$ существуют две возможности выбора знака компоненты скорости вдоль оси $y$ :

$$
V_{y}= \pm\left[2 c^{2}\left(E_{\perp}-U(x, y)-p_{x^{2}} c^{2} / 2 E_{\|}\right) / E_{\|}\right]^{1 / 2} \text {. }
$$

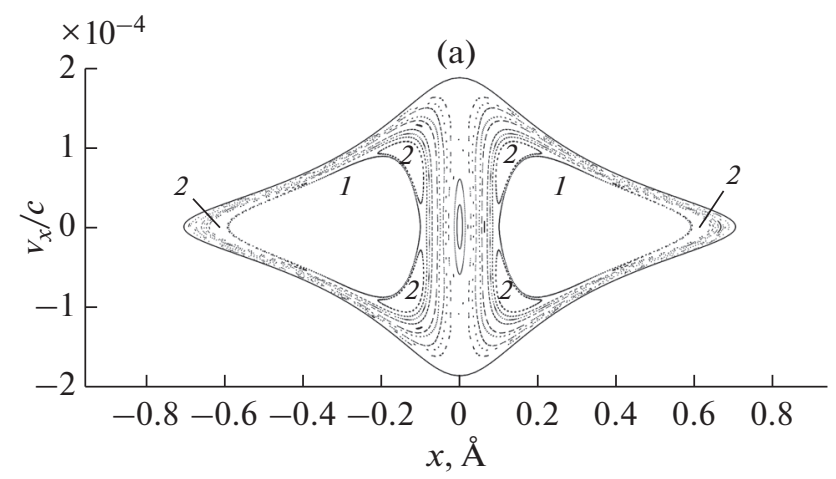

(б)

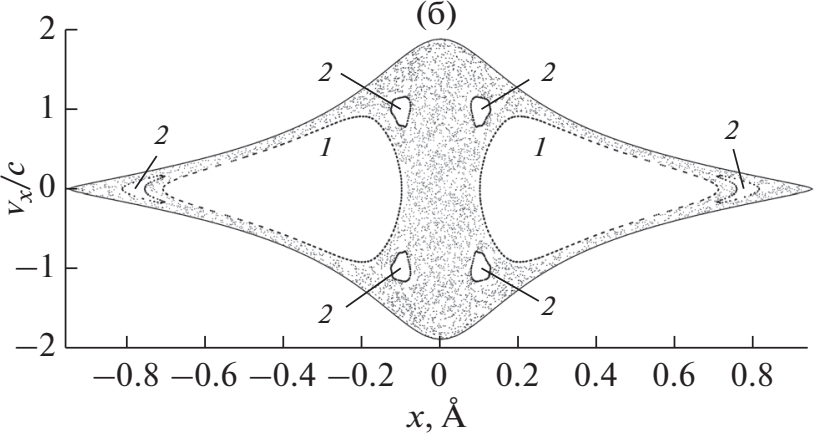

Рис. 2. Сечения Пуанкаре для $E_{\perp}=-14$ эВ (а) и $E_{\perp}=-12.0885$ эВ (б) электрона с энергией $E_{\|}=5$ ГэВ, движущегося в потенциале (10).

Однако, наличие этих двух возможностей уже учтено при переходе от (4) к (5), поэтому при реализации численного алгоритма мы всегда выбираем только один (положительный) знак.

Отметим, что в теории квантового хаоса исследуются флуктуации межуровневых расстояний относительного среднего значения 1/ $\rho$. Поскольку средняя плотность уровней энергии в потенциале вида (10) возрастает с ростом $E_{\perp}$, исходный массив уровней на исследуемом интервале подвергается процедуре развертывания [2, 4]. Новый массив уровней будет обладать единичной средней плотностью $(\rho=1)$.

\section{РЕЗУЛЬТАТЫ И ИХ ОБСУЖДЕНИЕ}

Построение сечений Пуанкаре показывает, что для состояний в глубине потенциальной ямы (10) динамика электрона полностью регулярна. Это обусловлено слабостью влияния на потенциал центральной атомной цепочки ее соседей, что приводит к сохранению при движении электрона, наряду с энергией $E_{\perp}$, проекции орбитального момента импульса на ось цепочки. С увеличением $E_{\perp}$ электрон получает возможность посещать при движении области на периферии элементарной ячейки, в которых влияние соседних цепочек приводит к значительному на- 


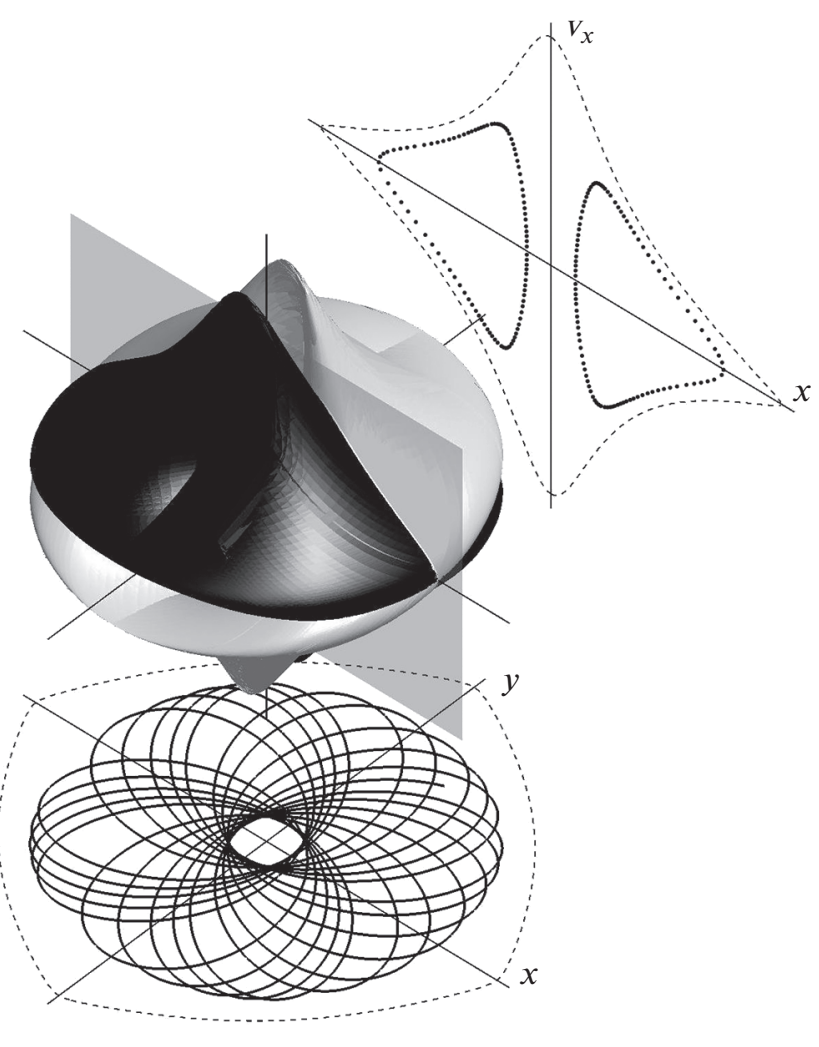

Рис. 3. Проекция на трехмерное пространство $\left(x, y, v_{x}\right)$ двух областей фазового пространства, соответствующих области типа 1 на сечении Пуанкаре (рис. 2б). Показаны также секущая плоскость $\left(x, v_{x}\right)$, сечение Пуанкаре и траектория электрона в плоскости $(x, y)$. Светлая область соответствует движению по орбите против часовой стрелки, темная - по часовой стрелке.

рушению аксиальной симметрии потенциала, следствием чего является хаотизация движения (начиная с энергии $E_{\perp}=-14$ эВ для электрона с $E_{\|}=5$ ГэВ).

Среди областей регулярного движения выделяется область 1 на рис. 2. Движение в ней близко к движению в центральном поле. Наличие этой области прослеживается во всем обсуждаемом интервале энергий поперечного движения, от -14 эВ (полностью регулярное движение) до -12 эВ (верхний край потенциальной ямы), причем в области $E_{\perp} \geq-12.8$ эВ вклад этой области становится определяющим (для более глубоких уровней имеются также регулярные торы, охватывающие на графиках Пуанкаре области типов 1 и 2).

Регулярность движения именно в этой области допускает простую и наглядную интерпретацию. Вблизи оси цепочки возмущающее влияние соседних цепочек пренебрежимо мало, что приводит к приблизительному сохранению орбиталь- ного момента импульса. Возникающий при этом центробежный барьер “не подпускает” частицу к тем областям, где сохранение момента импульса отсутствует и возникает хаотизация движения.

Оказывается, что вклад этой области в среднюю плотность уровней с хорошей точностью постоянен на всем обсуждаемом интервале, и составляет приблизительно 34\%. Подчеркнем, что результат Берри-Робника-Богомольного (3) был получен в предположении постоянства вклада областей регулярного движения в плотность уровней на всем рассматриваемом интервале. Таким образом, величина $\rho_{1}=0.34$ может служить нижней оценкой вклада областей регулярной динамики на интервале

$$
-12.82 \leq E_{\perp} \leq-12.08 \text { эB }
$$

на котором вклад этой области становится определяющим. Среднее значение вклада всех регулярных областей на этом интервале составляет приблизительно $\rho_{1}=0.43$.

Отметим, что области регулярного движения на сечении Пуанкаре каждого типа, например, типа 1 , соответствуют две симметричные области, не пересекающиеся в четырехмерном фазовом пространстве, соответствующие движению по данной орбите по и против часовой стрелки в плоскости $(x, y)$. Однако, проекции этих областей на трехмерное пространство $\left(x, y, v_{x}\right)$ будут пересекаться, как показано на рис. 3. При этом, согласно сказанному при обсуждении формулы (13), соответствующий вклад в плотность $\rho_{1}$ уровней, связанных с регулярным движением, будет определяться интегралом вида (5) только по одной из двух таких трехмерных областей.

Форма аналогичной трехмерной области типа 2 (соответствующей движению против часовой стрелки) показана на рис. 4.

Квазиклассическая плотность уровней энергии электрона с энергией $E_{\|}=5$ ГэВ, каналированного в направлении [100] кристалла кремния, рассчитанная по формуле (5) методом МонтеКарло, показана на рис. 5 сплошной линией, а оценка вклада в нее областей регулярного движения - точками. Погрешность этой оценки обусловлена как сложностью точного определения границ областей регулярного движения, так и сложностью учета вкладов небольших по объему областей регулярного движения, появление которых сопровождает разрушение инвариантных торов на границе областей регулярной и хаотической динамики.

На рис. 6 представлено распределение межуровневых расстояний для четырех типов невырожденных уровней энергии поперечного движения электрона с энергией продольного движения 5 ГэВ на интервале (14). Жирными линиями на рис. 6 обозначено распределение Берри-Робни- 


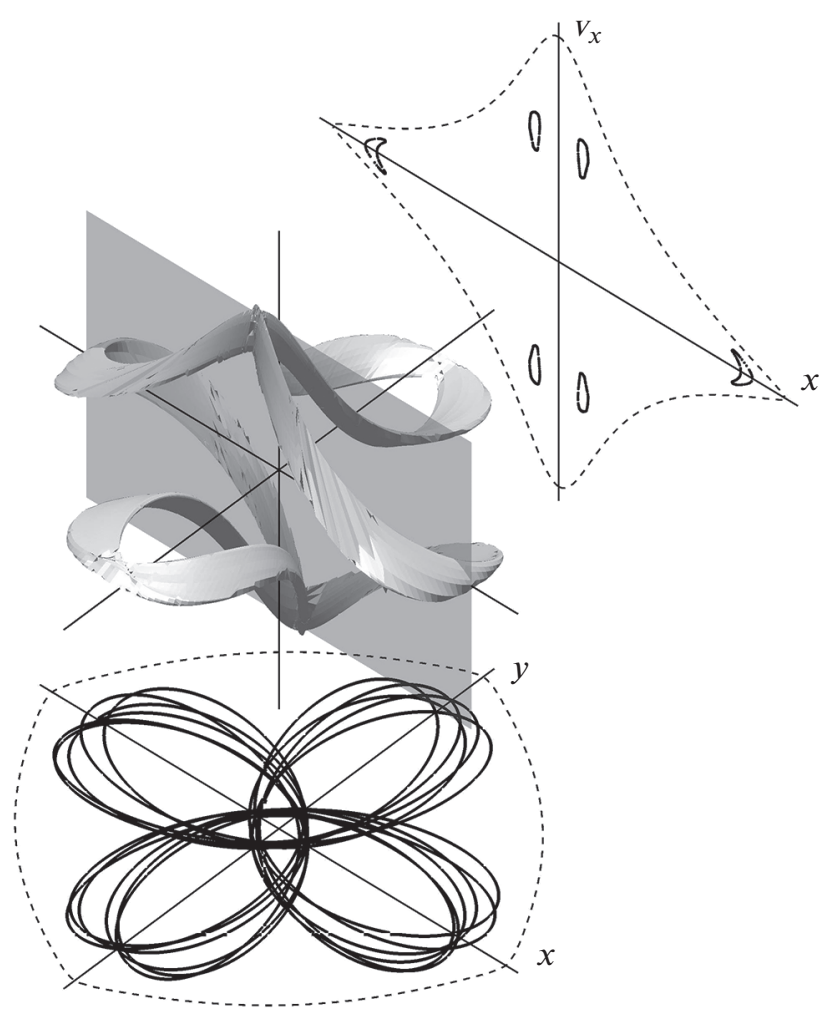

Рис. 4. Проекция на трехмерное пространство $\left(x, y, v_{x}\right)$ области фазового пространства типа 2 (соответствующей движению по орбите против часовой стрелки) для тех же условий, что и на рис. 3.

ка-Богомольного с $\rho_{1}=0.3428$ (жирная штриховая линия, величина $\chi^{2}$ в этом случае составляет 8.7225 ) и $\rho_{1}=0.4274$ (жирная сплошная линия, $\left.\chi^{2}=12.9508\right)$. В обоих случаях мы видим, что распределение Берри-Робника-Богомольного лучше описывает реальное распределение межуровневых расстояний по сравнению с распределениями (1) (тонкая штриховая линия, $\chi^{2}=17.9184$ ) и (2) (пунктирная линия, $\chi^{2}=30.8545$ ). Однако, фитирование по критерию максимального правдоподобия с величиной $\rho_{1}$ в качестве свободного параметра приводит к значению около 15\% (тонкая сплошная линия, $\left.\chi^{2}=3.5489\right)$, намного меньшему реального значения вклада области регулярных траекторий в плотность уровней на рассматриваемом интервале. Возникающее расхождение обусловлено тем, что распределение Берри-Робника-Богомольного предполагает, что хаотическая и регулярные области фазового пространства порождают две независимые последовательности уровней, пренебрегая корреляциями между последними.

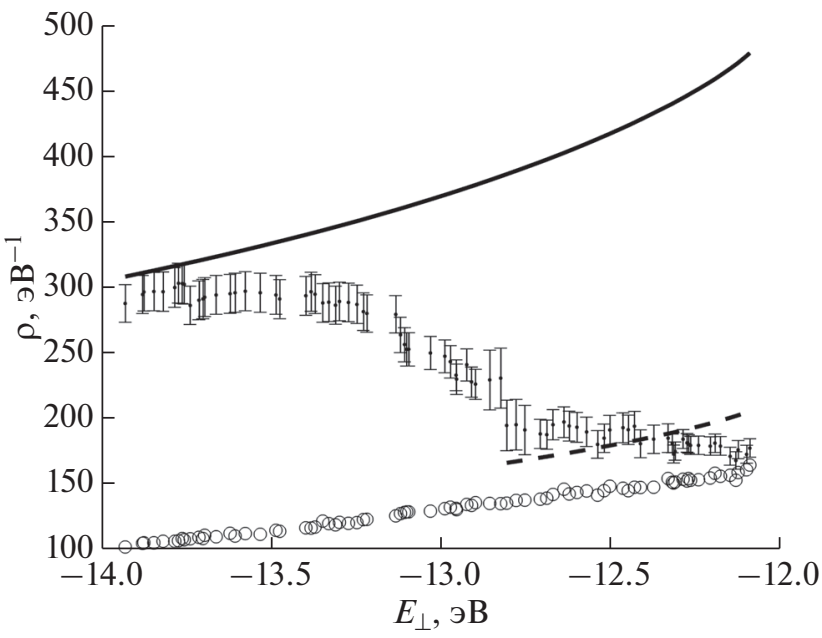

Рис. 5. Средняя плотность уровней энергии поперечного движения (сплошная линия) и вклад в нее областей типа 1 (кружки), а также суммарный вклад всех областей регулярного движения (точки; погрешность обусловлена трудностью точного определения границ областей регулярного движения). Штриховой линией отмечена средняя плотность уровней энергии, связанных с областями регулярного движения, на интервале (14).

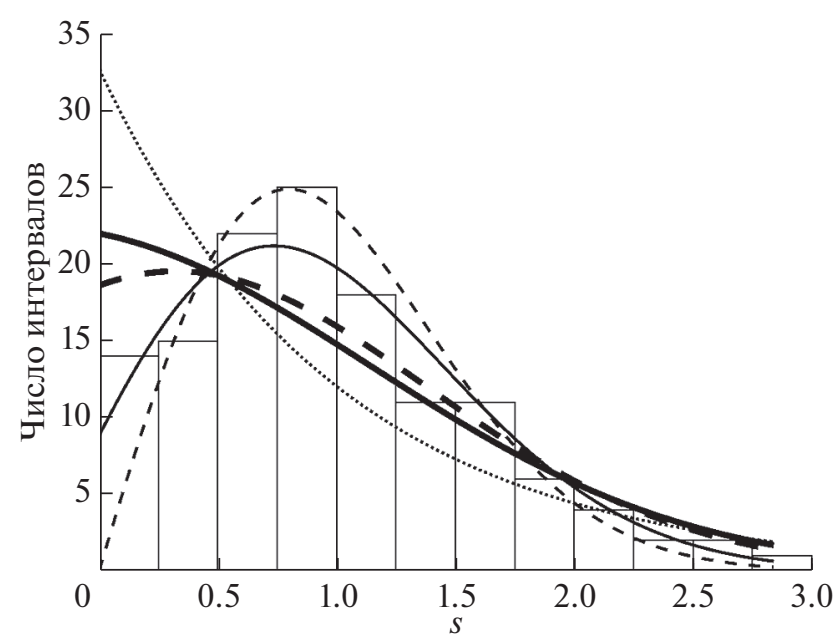

Рис. 6. Распределение межуровневых интервалов в области (14). Толстой сплошной линией показано распределение Берри-Робника-Богомольного для значения $\rho_{1}=0.4274$, толстой штриховой линией - то же для $\rho_{1}=0.3428$, тонкой сплошной линией - то же для значения $\rho_{1}=0.1450$, оцененного как результат фитирования по критерию максимального правдоподобия. Тонкая пунктирная линия - распределение (2), тонкая штриховая - распределение (1).

\section{ЗАКЛЮЧЕНИЕ}

В работе рассмотрено каналирование электронов с энергией 5 ГэВ вблизи цепочек [100] кристалла кремния. Показано, что вблизи верхнего 
края потенциальной ямы, образованной непрерывным потенциалом атомной цепочки, влияние соседних цепочек приводит к возникновению значительной области хаотической динамики в фазовом пространстве поперечного движения электрона.

В рамках квазиклассического приближения квантовой механики вычислена плотность уровней энергии поперечного движения электрона и определен вклад в эту величину сохранившихся в верхней части потенциальной ямы областей регулярного движения.

Значение относительного вклада областей регулярного движения в плотность уровней входит в качестве параметра в распределение БерриРобника-Богомольного, описывающее статистику межуровневых расстояний квантовой системы, чей классический аналог обладает частично регулярной, а частично хаотической динамикой. Установлено, что в рассматриваемом случае каналированного электрона распределение Берри-Робника-Богомольного лучше описывает статистические свойства невырожденных уровней энергии, нежели чистые распределения Пуассона и Вигнера. Однако, распределение Берри-Робника-Богомольного не учитывает ряд особенностей динамики системы. В связи с этим можно ожидать, что распределение Подольского-Нариманова [15], учитывающее влияние туннелирования, сопровождаемого хаосом, будет лучше описывать фактическое распределение уровней энергии поперечного движения электронов на малых межуровневых расстояниях.

\section{СПИСОК ЛИТЕРАТУРЫ}

1. Berry M.V. // Proceedings of the Royal Society A. 1987. V. 413. P. 183.

https://doi.org/10.1098/rspa.1987.0109
2. Bohigas O., Giannoni M.-J. // Lecture Notes in Physics. 1984. V. 209. P. 1-99.

3. Штокман Х.-Ю. Квантовый хаос. М.: ФИЗМАТЛИТ, 2004. $376 \mathrm{c}$.

4. Райхл Л.Е. Переход к хаосу в консервативных классических и квантовых системах. М.-Ижевск: РХД, 2008. $756 \mathrm{c}$.

5. Ахиезер А.И., Шульга Н.Ф. Электродинамика высоких энергий в веществе. М.: Наука, 1993. 344 с.

6. Ахиезер А.И., Шульга Н.Ф., Трутень В.И. и др. // УФН. 1995. Т. 165. № 10. С. 1165.

7. Шульга Н.Ф., Сыщенко В.В., Тарновский А.И., Исупов А.Ю. // Поверхность. Рентген., синхротр. и нейтрон. исслед. 2015. № 7. С. 72. https://doi.org/10.7868/S0207352815070197

8. Shul'ga N.F., Syshchenko V.V., Tarnovsky A.I., Isupov A.Yu. // Nucl. Instrum. Methods. B. 2016. V. 370. P. 1. https://doi.org/10.1016/j.nimb.2015.12.040

9. Shul'ga N.F., Syshchenko V.V., Tarnovsky A.I., Isupov A. Yu. // Journal of Physics: Conference Series. 2016. V. 732. P. 012028. https://doi.org/10.1088/1742-6596/732/1/012028

10. Berry M.V., Robnik M. // J. Phys. A.: Math. Gen. 1984. 17. P. 2413. https://doi.org/10.1088/0305-4470/17/12/013

11. Богомольный Е.В. // Письма ЖЭТФ. 1985. Т. 11. № 2. C. 55.

12. Feit M.D., Fleck J.A., Jr., Steiger A. // J. of Computational Physics. 1982. V. 47. P. 412.

13. Шульга Н.Ф., Сыщенко В.В., Нерябова В.С. // Поверхность. Рентген., синхротр. и нейтрон. исслед 2013. № 3. C. 91. https://doi.org/10.1134/S1027451013020183

14. Ландау Л.Д., Лифшии, Е.М. Теоретическая физика. В 10 томах. Т. 3. Квантовая механика. Нерелятивистская теория. М.: ФИЗМАТЛИТ, 2016. 800 с.

15. Podolskiy V.A., Narimanov E.E. // Phys. Lett. A. 2007. V. 362. P. 412.

https://doi.org/10.1016/j.physleta.2006.10.065

\title{
The Structure of the Regular Motion Domains in the Phase Space of the Channeling Electrons
}

\author{
V. V. Syshchenko, *, A. I. Tarnovsky' ${ }^{1}$, A. Yu. Isupov², I. I. Solovyev ${ }^{1}$ \\ ${ }^{1}$ Belgorod State University, Street Pobedy 69A, Belgorod, 308015 Russia \\ ${ }^{2}$ Laboratory of High Energy Physics, Joint Institute for Nuclear Research, Dubna, 141980 Russia \\ *e-mail:syshch@yandex.ru
}

\begin{abstract}
The electron's motion under axial channeling in [100] direction of a silicon crystal can be both regular and chaotic, depending on the initial conditions. The contribution of the domains of regular and chaotic dynamics into the semiclassical density of the electron's transverse motion energy levels is computed in the present paper. The quantities obtained are used as the parameters of Berry-Robnik distribution that describes the level spacing statistics in the case of co-existence of the regular and chaotic motion domains.
\end{abstract}

Keywords: regular dynamics, chaotic dynamics, quantum chaos, channeling, semiclassical approximation, level spacing statistics, Berry-Robnik distribution. 